\title{
Perfil Cognitivo y Psicolinguístico y su Relación con la Lectoescritura en un Preescolar con Síndrome de Down
}

\author{
Cognitive and Psycholinguistic Profile and its Relationship with Reading and Writing \\ in a Preschool with Down Syndrome
}

\author{
Maria Auxiliadora Bello ${ }^{1}$, Nieves Valencia Naranjo ${ }^{2}$, Juana María Ruiz Cano ${ }^{3}$ y David Sánchez \\ Teruel $^{4}$
}

\begin{abstract}
Resumen
El Síndrome de Down (SD) va a provocar que se manifiesten alteraciones en el desarrollo cognitivo que pueden afectar al proceso de adquisición y desarrollo de la lectoescritura. En el presente estudio se ha caracterizado el perfil cognitivo/psicolingüístico y el nivel de lectoescritura de un niño de 5 años con SD en comparación con un niño de desarrollo típico (DT). Además, se ha llevado a cabo un Programa de Lectoescritura con el niño SD. La hipótesis se basa en que las diferencias cognitivas presentes en SD con respecto al DT van a interferir en el desarrollo de la lectoescritura. Sin embargo, estas diferencias en lectoescritura pueden verse disminuidas por la participación en un programa de atención temprana (AT). Los resultados muestran cómo existe una diferencia a nivel cognitivo/lingüístico entre ambos participantes, sin embargo, estas diferencias son menores en el nivel de lectura y escritura alcanzado tras un programa de AT.
\end{abstract}

Palabras clave: Síndrome de Down, estudio de caso, atención temprana, perfil cognitivo, lectoescritura

\begin{abstract}
Down Syndrome (DS) will cause alterations in cognitive development. Thus, it is necessary to consider how reading and writing development there are individual determinants which can affect this process. This study assessed the cognitive and psycholinguistic profile and writing and reading level of a 5 year old child with DS compared with a typically developing child. The aims of the present study is know some cognitive differences present in the intellectual disability compared to typical development which could interfere with reading and writing development. However, these differences in reading and writing may be lower, among other things, with specific early intervention focused on reading and writing in children with DS. The findings evidence a difference in cognitive and language profile between participants, however, these differences are lower in reading and writing level after early intervention.
\end{abstract}

Keywords: Down Syndrome, case study, early care, cognitive profile, reading, writing

\footnotetext{
${ }^{1}$ Profesora del Departamento de Psicología de la Universidad de Jaén. Coordinadora del Centro de Atención Infantil Temprana Down Jaén. Paraje de las Lagunillas, s/n. 23071. Jáen, España. Tel.: 953260413. Correo:marobles@ujaen.es

2 Profesora titular del Departamento de Psicología de la Universidad de Jaén. Campus Las Lagunillas. Edificio Humanidades y Ciencias de la Educación C5, dependencia 122. Jaén, España. Correo: nnaranjo@ujaen.es

${ }^{3}$ Postgrado. Departamento de Psicología de la Universidad de Jaén. Paraje de las Lagunillas, s/n. 23071. Jáen, España. Tel.: 953260413. Correo: jmrc0019@red.ujaen.es

${ }^{4}$ Profesor del Departamento de Psicología de la Universidad de Córdoba. C/ San Alberto Magno s/n. 14071 Córdoba, España. Tel.: 9572189 23. Correo: dsteruel@uco.es
} 


\section{Introducción}

El Síndrome de Down y su trisomía regular (SD) es la cromosomopatía de etiología conocida más frecuente en neonatos a nivel mundial, que cursa con discapacidad intelectual en grado variable (Robles-Bello \& Sánchez-Teruel, 2011). El rendimiento intelectual en este grupo de personas varía desde valores en el cociente intelectual (CI) inferiores a 25-30 a superiores a 60 , en la minoría de los casos. No obstante, las puntuaciones medias de CI obtenidas a través de pruebas estandarizadas oscilan entre 40 y 55 (Robles-Bello, 2007). Los factores que influyen en este enorme rango son la edad, el ambiente del individuo (este se hace determinante) y los factores genéticos que determinan como serán sus estructuras cerebrales. Cuantas menos afecciones tanto a nivel estructural como funcional del sistema nervioso central presente la persona, mayor probabilidad habrá de que su desarrollo cognitivo sea mejor; así, también es razonable pensar que las mejorías en los diferentes aspectos neuropsicológicos gracias a los efectivos programas de intervención temprana puedan influir positivamente en la capacidad neuroquímica para aprender y retener nueva información (García, Portellano \& Díaz, 2010).

Además de su relación con la discapacidad intelectual, la trisomía regular parece conducir a un perfil neuropsicológico específico. El estudio de Galeote, Soto, Sebastián, Rey, \& Checa (2012) sugiere que el desarrollo del lenguaje constituye una de las áreas en las que los niños con SD presentan mayores dificultades. El perfil de habilidades a nivel lingüístico en $\mathrm{SD}$ es cuantitativa y cualitativamente distinto al perfil evolutivo al observado en niños con desarrollo típico (DT). Concretamente, un retraso específico en relación con otras áreas del desarrollo (ej. cognitiva, social) y que se acentúa en etapas posteriores del desarrollo así como disociaciones específicas entre componentes lingüísticos (ej. mayor desarrollo léxico frente al fonológico y/o morfosintáctico) o procesos lingüísticos (ej. mejores habilidades de comprensión que de producción, especialmente de la comprensión léxica frente a la sintáctica). Otras características en este perfil neuropsicológico en SD es la presencia de habilidades visoespaciales mejor preservadas y déficit importantes en el procesamiento de los aspectos perceptuales como el color (Valencia \& Robles-Bello, 2017); disfunción ejecutiva sobre todo en los procesos subregionales, como la memoria de trabajo para el material verbal (Edgin, Pennington, \& Mervis, 2010) así como un deterioro de la memoria episódica ligada a la edad avanzada (KrinskyMcHale, Kittler, Brown, Jenkins, \& Devenny, 2005).

De forma global, los resultados de distintos trabajos (Buckley \& Sacks, 2002; Buckley \& Perea, 2005; Calero, Robles-Bello, \& García, 2010) sugieren que cuando se compara la ejecución de personas con SD y controles, las mayores diferencias se relacionan, fundamentalmente, con la modalidad auditivoverbal, tanto en memoria como en procesamiento (ej. planificación verbal)

Las personas con SD desarrollan su inteligencia a lo largo de su vida, si se les ofrecen oportunidades para aprender desde el mismo nacimiento. De esta perspectiva surgen los programas de intervención en Atención Infantil Temprana (AIT) que potencian, para los niños con dificultades en general, y en SD en particular, un inicio en la Escuela Primaria en óptimas condiciones (Troncoso \& del Cerro, 2005;). Las áreas en las que normalmente se organiza un programa de intervención en AIT son la motora, comunicativa o del lenguaje, autonomía/social y cognitiva (Jenaro \& Gutiérrez-Bermejo, 2014; Robles-Bello \& Sánchez-Teruel, 2013). Cada una de ellas se compone de diversas subáreas de las cuales suelen derivarse los objetivos generales de los diversos programas. Más concretamente, dentro del área cognitiva, compuesta por las habilidades y capacidades de tipo conceptual, se incluyen las habilidades escolares (Buceta, 2011). En este tipo de programas se enmarcan aquellos dirigidos a la adquisición y desarrollo de la lectura y escritura, que entendemos es un requisito previo a trabajar muy importante en la integración y funcionamiento posterior en la sociedad.

El inicio del proceso de aprendizaje de la lectoescritura suele centrarse en la capacidad para decodificar y comprender el lenguaje escrito. Desde esta perspectiva, la Aproximación Simple de la Lectura (ASL- Simple View of Reading) de Gough \& Tummer (1986) sugiere que la 
comprensión lectora deriva de las habilidades de comprensión lingüística y de la decodificación eficiente del lenguaje escrito.

Torppa, Georgiou, Lerkkanen, Niemi y Poikkeus (2016) examinaron el potencial de aplicación de la ASL en el estudio de la comprensión lectora de niños preescolares con un DT y cuyo idioma materno, finlandés, muestra una transparencia ortográfica -grado de correspondencia en la aplicación de las reglas de conversión fonema-grafema- similar a la del español. Estos investigadores utilizando un procedimiento de análisis basado en ecuaciones estructurales, hallaron que las habilidades cognitivas relacionadas con la lectura (ej. conciencia fonológica, conocimiento de las letras, denominación rápida y vocabulario) y evaluadas en preescolar, predecían la comprensión de la lectura en los Grados 2 y 3 de forma indirecta a través de la comprensión lingüística y la fluidez en la lectura aunque de forma directa en el Grado 1. Otros resultados relevantes en relación con el Grado 1 son que la comprensión lectora correlaciona de forma importante con la comprensión auditiva $\mathrm{y}$, especialmente, con fluidez lectora y que las habilidades cognitivas son un importante predictor de la capacidad de decodificación y de la comprensión lectora alcanzada por los niños. En el Grado 2, la comprensión lectora era predicha por la fluidez lectora y la comprensión lingüística $(37 \%$ de la varianza) mientras que en el Grado 3 solo participaba en la predicción la comprensión lingüística. Una de las conclusiones de Torppa et al (2016) el que papel predictivo de la fluidez lectora en la comprensión lectora se reduce de forma importante tras el primer año de enseñanza formal (Grado 1) puesto que en sistemas transparentes (ej. finlandés, español) los niños automatizan con relativa facilidad la decodificación del lenguaje escrito permitiéndoles dirigir sus recursos cognitivos hacia la comprensión del material.

Los procesos implicados en la adquisición de la lectura en niños con SD son menos conocidos. La fluidez lectora, y su correlación con la comprensión lectora, parece especialmente difícil de lograr por niños con SD. En esta población, la estrategia usual de enseñanza es de tipo visual y de palabra completa intentando superar las dificultades fonológicas en este grupo y apoyándose en sus mejores habilidades de procesamiento visual (Snowling, Hulme, \& Mercer, 2002). Sin embargo, van Bysterveldt \& Gillon (2014) sugieren que esta aproximación más global no excluye que las experiencias adecuadas de lectura contribuyan a que los niños establezcan la relación entre las representaciones ortográficas y fonológicas del lenguaje. De acuerdo a su hipótesis, los niños con SD mostraban distinto grado de desarrollo en tareas de conciencia fonológica y en la lectura de palabras aunque el grupo de participantes, con edades comprendidas entre los 5 y los 14 años, mostraban una gran variabilidad en su ejecución. Estos investigadores también identificaron que la exactitud lectora siempre era superior a su comprensión lectora, pese a la mayor conciencia fonológica identificada en algunos de los niños. Así mismo, los niños con mejor conocimiento de la estructura fónica del lenguaje eran aquellos que también tenían mayor conocimiento del nombre de las letras.

En definitiva $\mathrm{y}$, tras lo descrito en este apartado, se hace preciso tener en cuenta cómo en el proceso de adquisición y desarrollo de la lectoescritura, es necesario atender a los determinantes individuales que pueden afectar a dicho proceso. En el caso de los niños con SD, presentan dificultades en algunas áreas del desarrollo, que de una $\mathrm{u}$ otra forma pueden obstaculizar el proceso de aprendizaje de la lectoescritura. En dicho proceso se encuentran implicadas capacidades como la atención y memoria, el esquema corporal, la organización espacio-temporal, la coordinación visomotriz y el lenguaje básico, entre otras (Bautista, 2002). Con los actuales programas de AT se puede favorecer la adquisición y desarrollo de habilidades de lectoescritura que puedan llevar al niño con SD más cerca del desarrollo típico.

Así pues, en la presente investigación se aborda el estudio del perfil cognitivo $y$ psicolingüístico en un preescolar con $\mathrm{SD}$ en comparación con un preescolar de desarrollo típico (DT) así como el nivel alcanzado en el proceso de lectoescritura de ambos. Se pretende conocer si existe relación entre los déficits cognitivos y psicolingüísticos que se encuentran en un niño con SD y el proceso de adquisición de la lectoescritura. Este propósito se puede 
operacionalizar en una serie de objetivos específicos tales como: 1) comprobar las diferencias existentes en el perfil cognitivo y psicolingüístico entre un niño con $\mathrm{SD}$ y un preescolar con desarrollo típico; 2) analizar si existen diferencias entre el nivel alcanzado en lectoescritura entre ambos y 3 ) establecer el nivel de relación que existe entre el perfil cognitivo/lingüístico y el nivel de lectoescritura, antes y después de un programa de AT. En definitiva se trata de evaluar la eficacia de un programa de intervención en la lectura y escritura en un niño con SD.

\section{Método}

\section{Participantes}

La muestra del presente estudio está compuesta por 2 niños españoles varones de 5 años, uno de ellos diagnosticado con SD sin ningún problema adicional de salud según sus informes de atención temprana. El otro participante no presenta ninguna alteración de salud y presenta desarrollo adecuado a su edad según su maestra (niño con desarrollo típico -DTen adelante). El nivel educativo es de preescolar de 5 años, estando ambos escolarizados desde los 3 años. El niño SD, además de su escolarización regular, recibe tratamiento en un Centro de Atención Infantil Temprana del Sur de España desde su nacimiento y, actualmente, continúa en el programa.

Se solicitó autorización escrita a ambos padres para incluir a los niños como participantes en el estudio. El consentimiento informado incluía detalles del estudio como el tipo pruebas aplicadas y objetivos que se perseguían en el estudio. Así mismo, constaba el cumplimiento de la Ley de Protección de Datos de Carácter Personal 15/1999. Además, este trabajo fue aprobado por el comité de bioética de la Universidad de Jaén.

\section{Medidas e instrumentos de evaluación e intervención}

La Escala de Inteligencia de Wechsler para preescolar y primaria -WPPSI- (Weschsler, 1967) es una batería formada por varios subtest de medida de los aspectos cualitativo y cuantitativo de la inteligencia general. El WPPSI se divide en dos partes: Verbal y Manipulativa. Entre los subtest que evalúan la inteligencia verbal se encuentran: información, vocabulario, aritmética, semejanzas, comprensión y frases (complementaria). Los subtest que evalúan la inteligencia manipulativa son: casa de los animales, figuras incompletas, laberintos, dibujo geométrico y cuadrados. Su administración es individual, aplicable a niños de 4 a $6 \frac{112}{2}$ años. La duración de la prueba puede ser variable, entre 60 y 90 minutos. La fiabilidad varía en función de la prueba y del grupo de edad; para la edad de 5-5 1/2 años el coeficiente de fiabilidad es de 0,93 (CI verbal y manipulativo) y 0,95 (CI total escala).

$\begin{array}{ccc}\text { El Cuestionario de } & \text { Madurez } \\ \text { Neuropsicológica Infantil } & \text {-CUMANIN- }\end{array}$
(Portellano, Mateos, \& Martínez, 2000) se administra de forma individual y su objetivo general es la evaluación neuropsicológica de niños en edad de 3 a 6 años ( 36 meses a 78 meses). La duración de la prueba es variable, oscilando entre 30 y 50 minutos. El CUMANIN contiene escalas principales (psicomotricidad, lenguaje articulatorio, lenguaje comprensivo, lenguaje expresivo, estructuración espacial, visopercepción, memoria icónica y ritmo) y auxiliares (atención, fluidez verbal, lectura, escritura y lateralidad). El nivel de fiabilidad alfa varía en función de la escala siendo .71 (psicomotridad), .92 (lenguaje articulatorio), .73 (lenguaje expresivo), $0,72 \quad$ (lenguaje comprensivo), .81 (estructuración espacial), .91 (visopercepción), .57 (memoria icónica) y .72 (ritmo).

La Prueba de Lenguaje Oral NavarraRevisada -PLON-R- (Aguinaga, Armentia, Fraile, Olangua, \& Uriz, 2004) es una prueba de administración individual de detección rápida o screening del desarrollo del lenguaje oral aplicable a niños de edad comprendida entre los 3 y 6 años. La duración es variable, oscilando entre los 10 y 12 minutos. El PLON-R consta de varios niveles lingüísticos: fonológico, morfológico, sintáctico, léxico, semántico o de uso comunicativo. El coeficiente de fiabilidad en 5 años y para cada nivel es de .91 (fonología), .43 (morfología-sintaxis), $\quad .87 \quad$ (forma), $\quad .54$ (contenido), .13 (uso) y .76 (total PLON-R).

Programa de atención temprana para la enseñanza de la lectura y escritura en Síndrome de Down (Troncoso \& del Cerro, 2005). Se trata 
de un método de lectura y escritura para alumnos con SD. El programa está estructurado facilitando su función de guía para los profesionales que trabajan en el campo de la atención temprana aunque también ha sido utilizado por profesionales de escuelas infantiles y familias.

El programa describe las secuencias que hay que seguir y los errores que hay que evitar en el proceso de enseñanza-aprendizaje de la lectura y escritura. Consta de múltiples ejemplos e ilustraciones que tratan de ayudar a los padres y profesionales durante este proceso. El método está estructurado y explicado teniendo en cuenta la situación ideal, que sería la de un niño de 3 a 5 años de edad, que ha participado en un programa de atención temprana en el que ha progresado adecuadamente en sus facultades perceptivas y manipulativas. No obstante, si el alumno tiene más edad, se elegirán las actividades y los materiales apropiados a su nivel de madurez y destrezas (ver programa completo en el enlace http://www.down21materialdidactico.org/librolect ura/Libro/index.html).

El aprendizaje de la lectura de los alumnos/as con SD debe comenzar con una lectura global de las palabras con apoyo de una imagen, retirando progresivamente el apoyo visual. Superada esta etapa, se incrementa progresivamente el número de palabras que lee, tomando el siguiente orden: se comienza con los sustantivos de una y dos sílabas y después se pasa a la lectura de los verbos. En tercer lugar se pasa a la formación de frases con los sustantivos y verbos que conoce asociados a situaciones reales de su vida cotidiana. Gradualmente se irá retirando el modelo presentado hasta que logre formar otras frases con idéntica estructura gramatical de forma autónoma. Cuando ha ido adquiriendo un número determinado de palabras acompañadas de su artículo, siempre antes de comenzar la fase analítica, es conveniente que aprenda a discriminar los artículos. Por último, el inicio de la fase analítica debe hacerse progresivamente, comenzando por los sustantivos trabajados (Angulo et al., 2008; Buckley \& Perea, 2005; Troncoso \& del Cerro, 2005).

Por otra parte el inicio de la escritura no tiene por qué ser simultáneo al de la lectura. El proceso de la adquisición de esta habilidad es más complejo que el de la lectura y debe tener en cuenta las habilidades grafomotrices del niño/a (Buckley \& Perea, 2005).Este proceso deberá iniciarse utilizando materiales alternativos que disminuirán las dificultades psicomotrices del niño/a en estos inicios. Así, trazos en el aire, pizarras, bandejas de arena o harina, etc., ayudarán en un primer momento a mejorar la precisión de los movimientos. Posteriormente, se podrá utilizar engrosadores del lápiz como adaptación para facilitar la prensión del útil escritor. En el aprendizaje de la escritura se comienza escribiendo su nombre, que ya identificado y leído, puede escribirlo. Después de su nombre, se puede continuar con su contexto más cercano (mamá/papá), valorando su trazo libre para reproducir lo que ya lee. Cuando se ha iniciado la actividad de escribir debe tener una continuidad, partiendo de lo conocido a lo desconocido. Una vez que ha adquirido el trazo de las grafías, resultan útiles algunas estrategias para el dominio de su escritura como realizar preguntas sobre lo que ha escrito (Angulo et al., 2008).

En general, este programa pretende adelantarse en el tiempo y facilitar el aprendizaje de la lectura y escritura a alumnos con discapacidad intelectual. Según Troncoso \& del Cerro (2005), es un método que ha demostrado su eficacia, es adecuado para lograr un aprendizaje grato, y con él se consiguen buenos resultados de comprensión, fluidez y afición lectoras. Los autores garantizan el éxito si se trabaja con constancia, si no se abandona el programa pronto y si se introducen las adaptaciones convenientes en cada momento del proceso enseñanzaaprendizaje.

\section{Diseño}

Se trata un estudio de caso único (Buela-Casal \& Sierra, 2002; Montero \& León, 2002), un caso clínico donde se aplica tratamiento temprano de lectura y escritura durante 6 meses en un Centro de Atención Infantil Temprana coordinado con los padres y el colegio. Se evalúa el perfil cognitivo y psicolingüístico así como el nivel de lectoescritura antes y después de la aplicación de un programa de tratamiento de la lectoescritura en un niño con $\mathrm{SD}$, comparándose ambas situaciones con un niño con DT. 


\section{Procedimiento}

Tras recibir la valoración positiva del Comité de Bioética de la Universidad de Jaén, se contactó con las familias para solicitar su colaboración informando en detalle las características del estudio y firmar el consentimiento correspondiente. Posteriormente se procedió a la evaluación en los dos casos.

El proceso de evaluación neuropsicológica fue llevado a cabo por una profesional graduada en psicología y estudiante del máster en Psicología General Sanitaria (PGS). La administración de las diferentes pruebas de evaluación se realizó en un Centro de Atención Infantil Temprana, en una sala adecuada para dicho proceso en dos momentos temporales (fase pre-post tratamiento). El número de sesiones fueron de 3 a 4 sesiones para cada participante (evaluación pre y post) con una duración variable, en función de la prueba administrada, oscilando desde 15 minutos hasta 90 minutos. Se administró de forma individual y en diferentes sesiones y de forma contrabalanceada la Escala de Inteligencia de Wechsler para preescolar y primaria - WPPSI -, el Cuestionario de Madurez Neuropsicológica Infantil - CUMANIN - y la Prueba de Lenguaje Oral Navarra-Revisada - PLON-R -.

En la fase de tratamiento se realizó el programa de lectura y escritura (Troncoso \& del Cerro, 2005) durante 6 meses. Por tanto se llevó a cabo un programa individual con un preescolar con SD, ajustado a su edad, nivel y necesidades. Las sesiones de tratamiento fueron impartidas individualmente con una duración aproximada de 1 hora semanal y llevadas a cabo utilizando recursos variados y metodología adecuada a cada área y objetivo. El objetivo principal fue dirigido a potenciar las áreas de aprendizaje de lectura y escritura. Tras la fase de tratamiento se llevó a cabo una fase post-tratamiento donde se volvieron a administrar las pruebas de evaluación a ambos participantes para comprobar los cambios existentes en las distintas pruebas cognitivas y lingüísticas y en el desarrollo de la lectoescritura.

El programa de Atención Infantil Temprana utilizado se refiere al Programa para la Estimulación del Desarrollo Infantil-PEDI de Zulueta y Mollá (2006): Este programa para la estimulación del desarrollo y presenta, ordenadas evolutivamente en un inventario, las principales adquisiciones del desarrollo del niño a lo largo de sus primeros años. El programa diseñado por estas autoras incluye: inventario del desarrollo, fichas de estimulación en las áreas motora, perceptivocognoscitiva, de lenguaje y social, y material didáctico. La información obtenida en la anamnesis, junto con la derivada de la aplicación de las pruebas y escalas de desarrollo constituye la base para la programación y puesta en marcha de los objetivos de intervención. Esta propuesta consiste en atender dentro del programa las necesidades de aprendizaje en las que el niño presenta los mayores déficits. Por tanto, en el PEDI se contempla la necesidad de establecer un programa individual con cada sujeto, ajustado a su edad, nivel y necesidades del niño dirigido a potenciar las áreas cognitiva, motora, autonomía y de comunicación. Asimismo, enfatiza la participación activa de la familia durante todo el proceso de intervención. Las sesiones son impartidas individualmente con una duración de 45 minutos y llevadas con recursos variados y metodología adecuada a cada niño.

\section{Resultados}

El primer objetivo era comprobar posibles diferencias existentes en el perfil cognitivo y psicolingüístico entre un niño con $\mathrm{SD}$ y un preescolar con DT. La Tabla 1 recoge los resultados obtenidos en relación con prueba CUMANIN. Los resultados en esta prueba indican que existen diferencias entre las puntuaciones de ambos participantes en todas las pruebas que examinan el nivel de Desarrollo Verbal (DV) y nivel de Desarrollo No Verbal (DNV) así como en el índice de Desarrollo Global (DG). Estas diferencias se presentan tanto en la fase inicial de evaluación (fase Pre) como a los 6 meses (fase Post). En ambas fases, las puntuaciones obtenidas por el niño con SD, se encuentran por debajo de lo normativo (puntuaciones directas correspondientes a un $\mathrm{Pc}<50$ ), mientras que para el DT las puntuaciones se encuentran dentro del rango normativo (puntuaciones directas correspondientes a un $\mathrm{Pc}>50$ ) a excepción de la escala DV dónde la puntuación directa se encontraría por debajo de lo normativo (puntuación directa correspondiente a un Pc<50). Así mismo, se pueden observar que en todas las 
Tabla 1. Puntuaciones Directas y CD en el Cuestionario de Madurez Neuropsicológica Infantil (CUMANIN)

\begin{tabular}{lcccccc}
\hline & \multicolumn{3}{c}{ Puntuaciones SD } & \multicolumn{3}{c}{ Puntuaciones DT } \\
Subescalas & PRE & POST & Ganancia & PRE & POST & Ganancia \\
\hline Lenguaje Articulatorio (S1) & 6 & 7 & 1 & 11 & 12 & 1 \\
Lenguaje Expresivo (S2) & 0 & 0 & 0 & 3 & 3 & 0 \\
Lenguaje Comprensivo (S3) & 2 & 3 & 1 & 4 & 5 & 1 \\
Desarrollo Verbal (E1) & 8 & 10 & 2 & 18 & 20 & 2 \\
Psicomotricidad (S4) & 3 & 4 & 1 & 10 & 10 & 0 \\
Visopercepción (S5) & 6 & 7 & 1 & 10 & 12 & 2 \\
Memoria Icónica (S6) & 1 & 2 & 1 & 9 & 9 & 0 \\
Ritmo (S7) & 0 & 1 & 1 & 2 & 3 & 1 \\
Estructuración Espacial (S8) & 6 & 6 & 0 & 9 & 10 & 1 \\
Desarrollo No Verbal (E2) & 16 & 20 & 4 & 40 & 44 & 4 \\
Atención (EA) & 8 & 10 & 2 & 12 & 13 & 1 \\
Lateralidad & $\mathrm{D}$ & $\mathrm{D}$ & - & $\mathrm{D}$ & $\mathrm{D}$ & - \\
Desarrollo Global (E3) & 24 & 30 & 6 & 58 & 64 & 6 \\
Cociente de Desarrollo (CD) & 75 & 75 & 0 & 107 & 110 & 3 \\
Lectura & 3 & 7 & 4 & 6 & 7 & 1 \\
Escritura & 0 & 2 & 2 & 4 & 6 & 2 \\
\hline
\end{tabular}

*La referencia de cada escala (E) y subescala (S) es utilizada para la representación gráfica en la Figura 1.

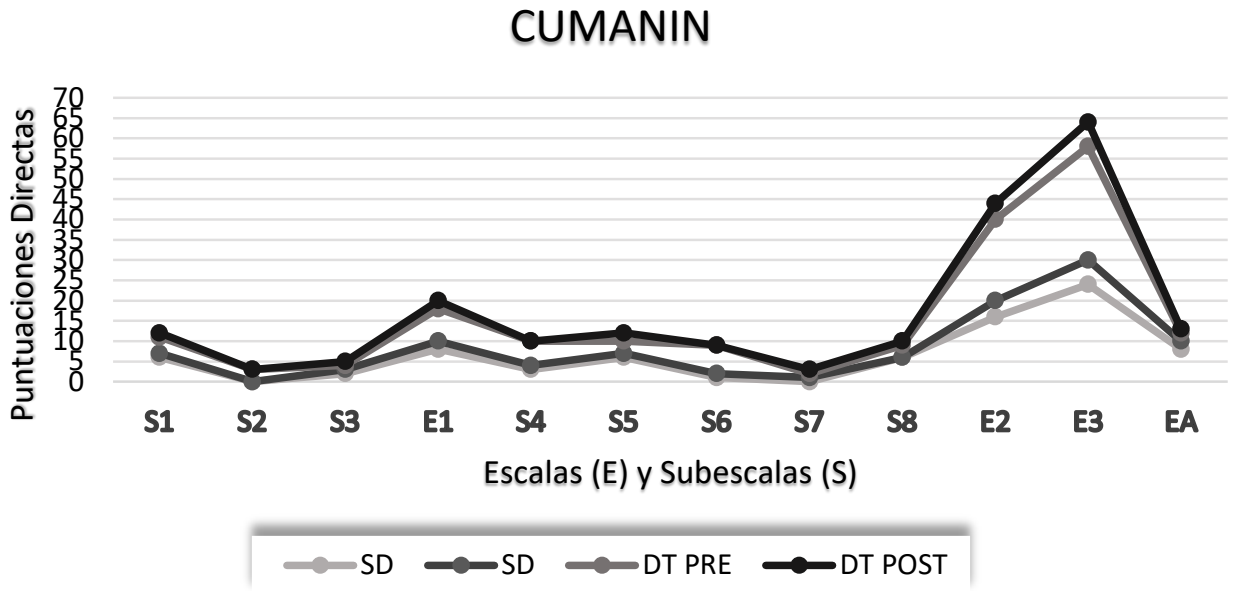

Figura 1. Puntuaciones Directas del Cuestionario de Madurez Neuropsicológica Infantil (CUMANIN) de ambos participantes en la situación pre y post tratamiento

escalas se produce una puntuación de ganancia $o$, al menos, un mantenimiento de la puntuación en la situación post con respecto a la situación pre. Tanto en el SD como en el DT existe una puntuación de ganancia, sin embargo, en el SD las puntuaciones siguen siendo considerablemente inferiores a las del DT.

Por último, el Cociente de Desarrollo (CD) final derivado de la prueba muestra las diferencias existentes entre ambos participantes siendo de 75 (en la situación post) para el SD y de 110 (en la situación post) para el DT.

Los resultados correspondientes a la prueba CUMANIN tanto para el niño con SD como para el niño con DT en las fases pre y post pueden observarse en la Figura 1 y Tabla 1.

Con respecto a la prueba de inteligencia administrada (WPPSI) los resultados obtenidos para ambos participantes antes y después del tratamiento se muestran en la Tabla 2. Existen diferencias en las puntuaciones directas en la mayoría de las subpruebas del WPPSI entre el SD $y$ el DT en la fase pre-tratamiento (tanto subpruebas verbales como manipulativas). Estos resultados se ven reflejados en el CI verbal, siendo éste diferente en 45 puntos, con respecto al DT; así mismo en el CI manipulativo existe una diferencia de 51 puntos, con respecto al DT; por 
Tabla 2. Puntuaciones Directas y CI Escala de Inteligencia de Wechsler para Preescolar y Primaria (WPPSI) y puntuaciones directas en la Prueba de Lenguaje Oral Navarra-Revisada (PLON-R)

\begin{tabular}{lcccccc}
\hline & \multicolumn{3}{c}{ Puntuaciones SD } & \multicolumn{3}{c}{ Puntuaciones DT } \\
Subpruebas & PRE & POST & Ganancia & PRE & POST & Ganancia \\
\hline Información (S1) & 9 & 10 & 1 & 9 & 9 & 0 \\
Vocabulario (S2) & 6 & 8 & 2 & 23 & 26 & 3 \\
Aritmética (S3) & 7 & 9 & 2 & 15 & 16 & 1 \\
Semejanzas (S4) & 5 & 5 & 0 & 12 & 13 & 1 \\
Comprensión (S5) & 4 & 4 & 0 & 18 & 19 & 1 \\
(Frases) (SA) & 1 & 1 & 0 & 11 & 11 & 0 \\
Casa de los animales (S6) & 10 & 10 & 0 & 44 & 46 & 2 \\
Figuras incompletas (S7) & 7 & 8 & 1 & 18 & 18 & 0 \\
Laberintos (S8) & 5 & 5 & 0 & 20 & 21 & 1 \\
Dibujo geométrico (S9) & 6 & 6 & 0 & 17 & 17 & 0 \\
Cuadrados (S10) & 5 & 8 & 3 & 15 & 15 & 0 \\
CI Verbal & 59 & 65 & 6 & 104 & 110 & 6 \\
CI Manipulativo & 63 & 66 & 3 & 114 & 119 & 5 \\
CI total & 54 & 59 & 5 & 111 & 117 & 6 \\
PLON-R & & & & & & \\
Forma & & & & & & \\
Contenido & 0 & 0 & 0 & 4 & 4 & 0 \\
Uso & 4 & 4 & 0 & 5 & 6 & 1 \\
TOTAL & 0 & 1 & 1 & 3 & 3 & 0 \\
\hline & 4 & 5 & 1 & 12 & 13 & 1 \\
\hline
\end{tabular}

\section{WPPSI}

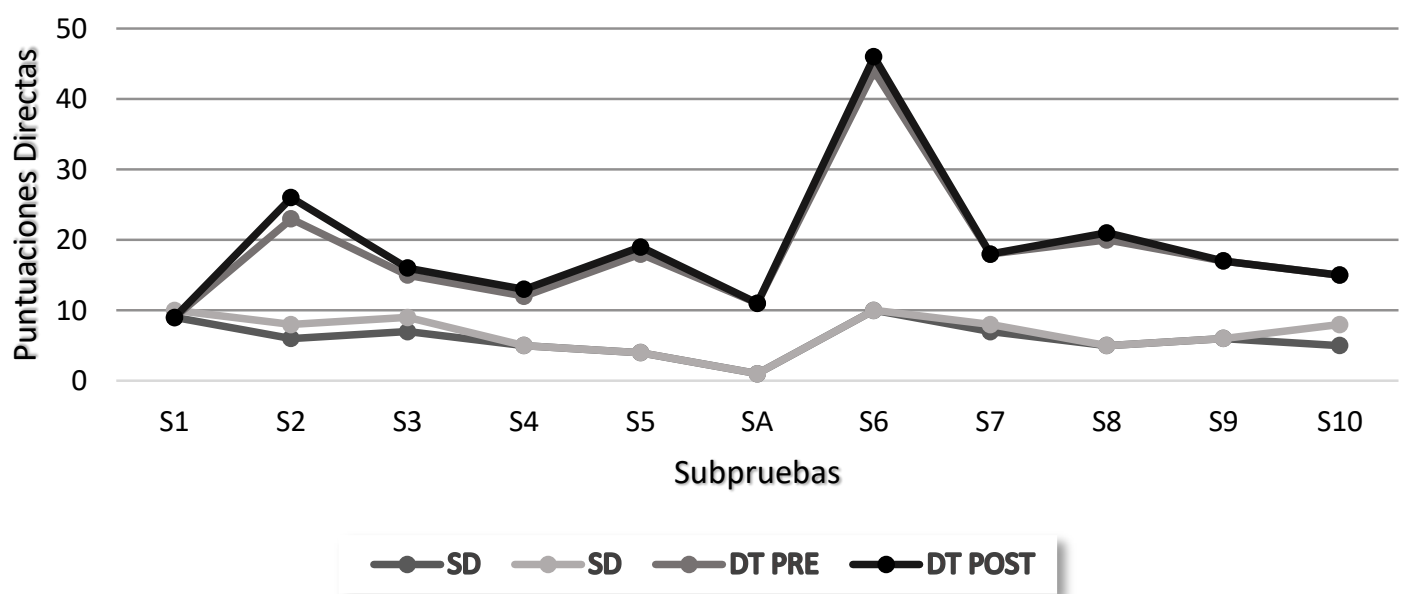

Figura 2. Puntuaciones Directas de las Subpruebas de la Escala de Inteligencia de Wechsler para Preescolar y Primaria (WPPSI) de ambos participantes en la situación pre y post tratamiento

último, en el CI total la diferencia entre el SD y el DT es de 57 puntos. Estas diferencias en las puntuaciones de las subpruebas y CI se mantienen en la fase post-tratamiento. Así se pueden observar diferencias entre el SD y el DT en la mayoría de las subpruebas del WPPSI (tanto subpruebas verbales como manipulativas) en la fase post-tratamiento. Con respecto al CI verbal, existe una diferencia de 45 puntos con respecto al DT; de igual manera, para el CI manipulativo la diferencia es de 53 puntos y, finalmente, para el CI total, en la fase post, la diferencia es de 58 puntos con respecto al DT.

Según la clasificación de la inteligencia a partir 


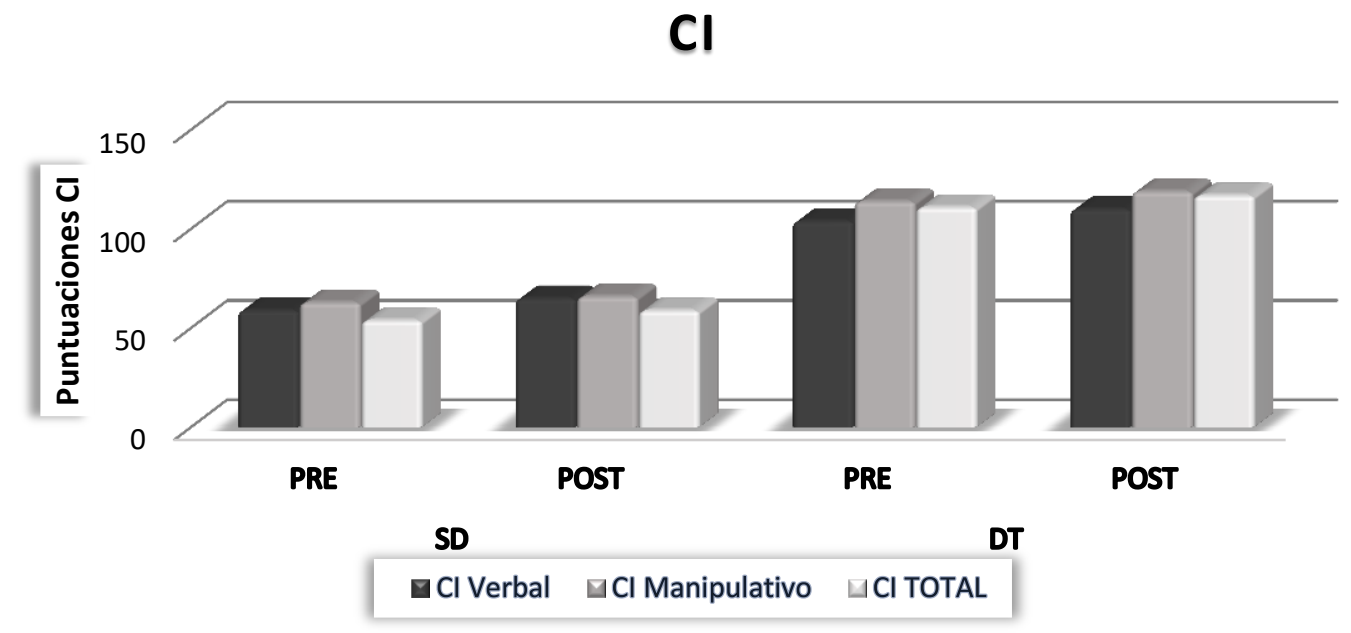

Figura 3. Puntuaciones CI de la Escala de Inteligencia de Wechsler para Preescolar y Primaria (WPPSI) para ambos participantes en la situación pre y post tratamiento

de los CI totales de la Escala de Inteligencia de Wechsler para Preescolar y Primaria (WPPSI), un CI correspondiente a 69 o menor, sería clasificado como deficiente (puntuaciones CI obtenidas para el participante con SD tanto en la situación pre como post). Por otro lado una puntuación comprendida entre 110-119 sería clasificada como normal-alto (puntuación obtenida para el participante con DT tanto en la situación pre como post).

La ganancia de puntuación para el SD en la fase post-tratamiento con respecto a la fase previa al tratamiento ha sido de 5 puntos para el CI total, mientras que para el DT la ganancia ha sido de 6 puntos para el CI total. A pesar de que ambos participantes obtienen una ganancia de puntuación en la segunda fase de evaluación, la puntuación del SD sigue estando por debajo de la puntuación obtenida por el participante con DT.

Los resultados correspondientes a la prueba WPPSI tanto para el niño con SD como para el niño con DT en las fases pre y post pueden observarse representados gráficamente en las Figuras 2 y 3.

Siguiendo con el primer objetivo, el resultado del perfil psicolingüístico obtenido de la Prueba de Lenguaje Oral Navarra-Revisada (PLON-R) (ver Tabla 2) muestra diferencias entre ambos participantes. En primer lugar, en la escala "Forma" se obtiene una diferencia entre el SD y el DT de 4 puntos, tanto en la situación pre como post-tratamiento. Dicha puntuación, según los criterios para la determinación del punto de corte de la prueba PLON-R, se sitúa por debajo de la media considerándose de déficit para el participante con $\mathrm{SD}(\mathrm{PD}=0-2)$, mientras que para el DT se situaría dentro de la puntuación entre la media y una desviación típica por debajo de la media considerándose que es necesario mejorar $(\mathrm{PD}=3-4)$. En segundo lugar, para la escala "Contenido" se obtiene una diferencia entre el SD y el DT de 1 y 2 puntos, en la situación pre y post, respectivamente. Así situaría al SD dentro de la puntuación considerada de necesita mejorar $(\mathrm{PD}=4)$, mientras que el DT se encontraría en la puntuación normal $(\mathrm{PD}=6)$. Finalmente, en la escala "Uso", existe una diferencia entre el SD y el DT de 3 y 2 puntos en la situación pre y post, respectivamente. De esta manera, la puntuación del SD lo sitúa dentro del rango de déficit $(\mathrm{PD}=0$ 1), mientras que para el DT la puntuación correspondería al rango de normalidad $(\mathrm{PD}=3)$.

Finalmente, la puntuación total de la prueba PLON-R sitúa al participante con SD en el rango déficit $(\mathrm{PD}=0-8)$, tanto en la fase pre como post, mientras que la puntuación del participante con DT se incluiría dentro del rango de normalidad $(\mathrm{PD}=11-14)$. La ganancia de la fase post con respecto a la fase pre es de 1 punto para cada participante en la puntuación total de la prueba. Sin embargo, sigue siendo considerablemente inferior la puntuación del SD con respecto al DT.

Los resultados correspondientes a la prueba PLON-R tanto para el niño con SD como para el niño con DT en las fases pre y post pueden observarse representados gráficamente en la Figura 4. 


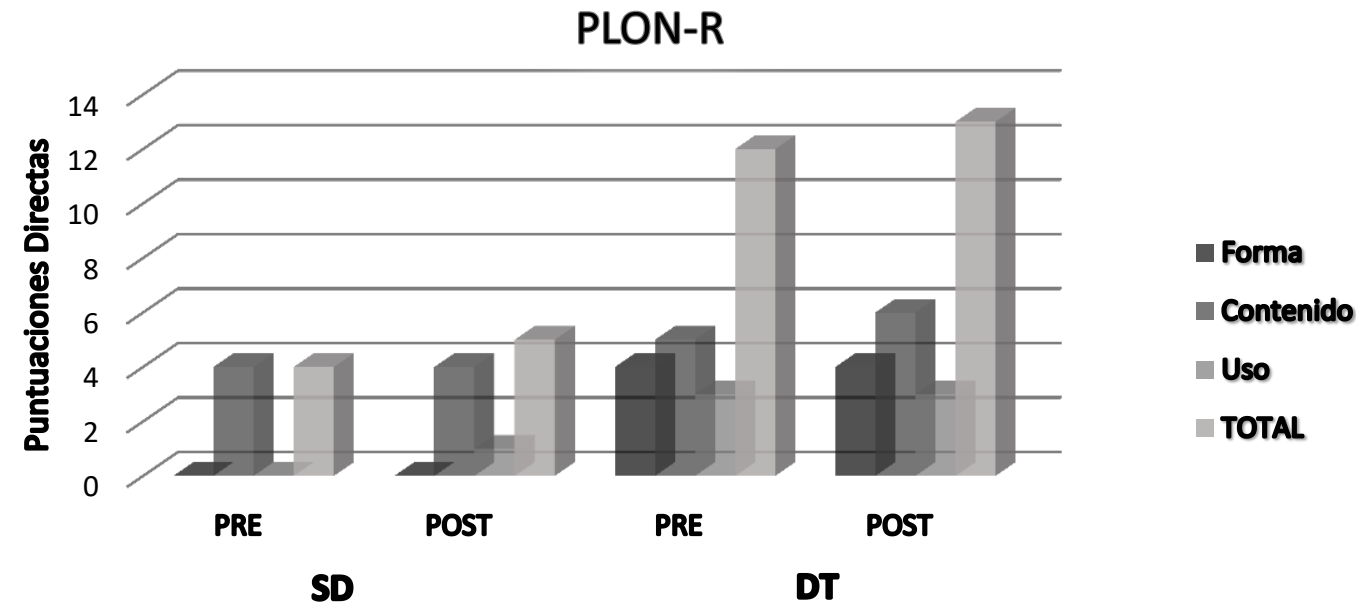

Figura 4. Puntuaciones directas de la Prueba de Lenguaje Oral Navarra-Revisada (PLON-R) para ambos participantes en la situación pre y post tratamiento

\section{CUMANIN. Lectura y Escritura.}

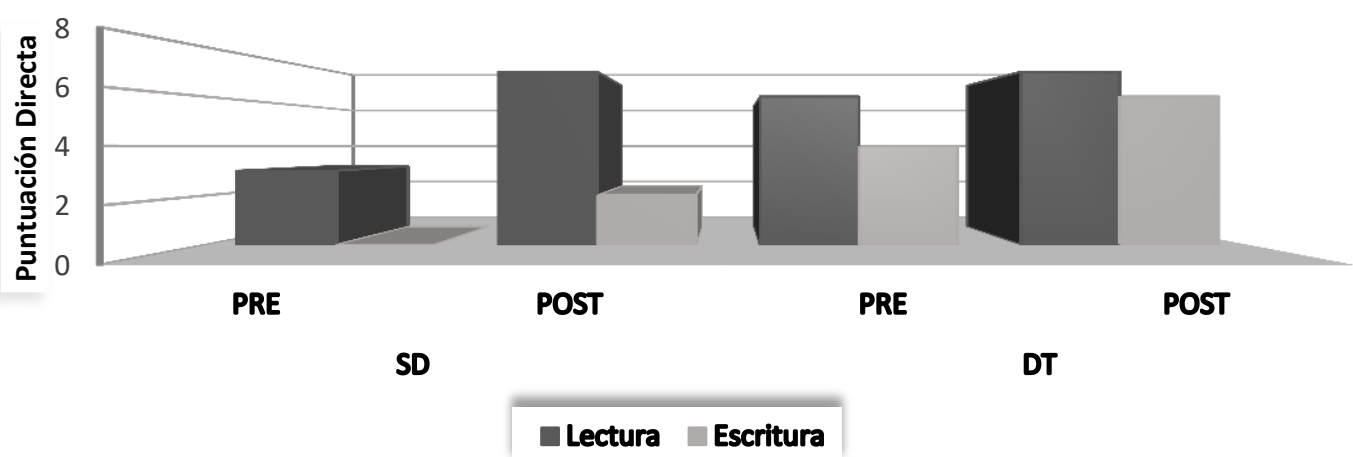

Figura 5. Puntuaciones directas de las subescalas Lectura y Escritura de la prueba CUMANIN para ambos participantes en la situación pre y post tratamiento

Con respecto al segundo objetivo de este estudio en el que se pretende observar si existen diferencias entre el participante con SD y el participante con DT en lectura y escritura, los resultados se muestran en la Tabla 1. La evaluación, realizada a través de las subescalas lectura y escritura de la prueba CUMANIN, muestra diferencias principalmente en escritura en la fase pre (4 puntos de diferencia), situando al participante con SD en una puntuación directa por debajo de la media $(\mathrm{PC}<50)$, mientras que el participante con DT obtiene una puntuación correspondiente a la media, adecuado a su nivel y grupo de edad (PC>50).

En el caso de la lectura la diferencia en la fase pre-tratamiento del SD con respecto al DT es de 3 puntos, aunque ambos se encuentran dentro del rango de normalidad ( $\mathrm{PC}>50$ ), siendo aun así la puntuación directa del participante com SD considerablemente menor que la puntuación del DT.

Tras el tratamiento de AT enfocado en lectura y escritura, fase post-tratamiento, se pueden observar los cambios en lectura y escritura del niño con SD. En el caso del niño con SD, tras la intervención en AT y el paso del tiempo, se observa una ganancia en las puntuaciones de lectura de 4 puntos $(\mathrm{PC}>50)$, siendo la ganancia en escritura de 2 puntos $(\mathrm{PC}<50)$. En el caso del niño con DT, el cuál no ha estado en el programa de AT, se observan también cambios debidos, principalmente al paso del tiempo, en lectura y escritura de 1 y 2 puntos, respectivamente. Por tanto, la ganancia ha sido principalmente destacable en la subescala de lectura en el participante con $\mathrm{SD}$, igualándose a la puntuación directa del participante con DT en la fase posttratamiento, tras el programa de lectura y escritura. 


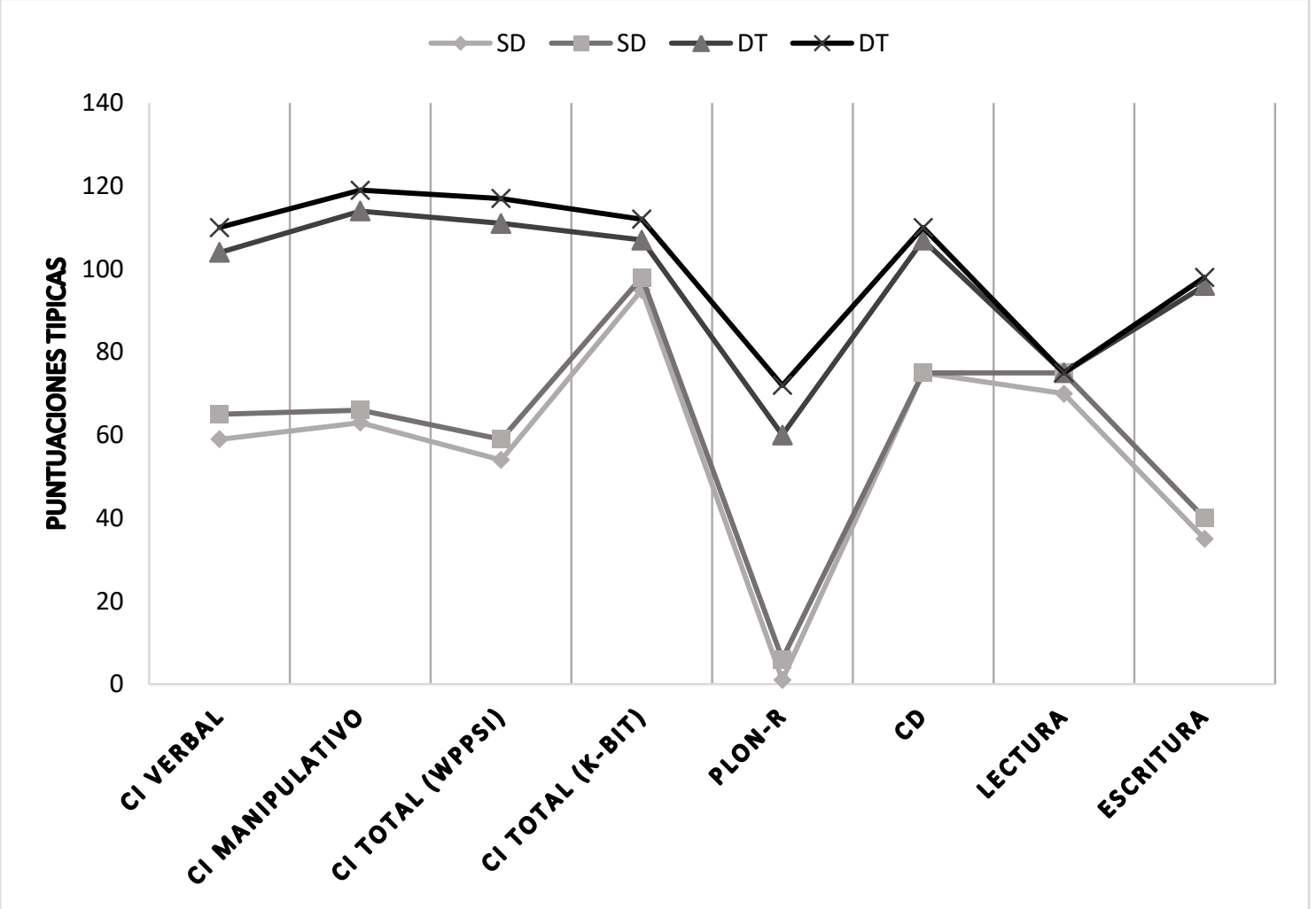

Figura 6. Puntuaciones típicas de todas las pruebas del perfil cognitivo y psicolingüístico de ambos participantes en la situación pre y post tratamiento

Los resultados correspondientes a las subpruebas de lectura y escritura de la prueba CUMANIN tanto para el niño con SD como para el niño con DT en las fases pre y post pueden observarse representados gráficamente en la Figura 5.

Por último, con respecto al tercer objetivo de este estudio que pretende establecer el nivel de relación que existe entre el perfil cognitivo/lingüístico y el nivel de lectoescritura, antes y después de un programa de AT, los resultados, en puntuaciones típicas, se muestran en la figura 6. Así, se puede observar cómo, tanto a nivel cognitivo (CD y CI verbal/manipulativo/total) como lingüístico (PLON-R) se obtienen puntuaciones del SD inferiores a las encontradas en el DT, tanto en la situación pre como post tratamiento. De igual manera, en escritura las puntuaciones son diferentes entre el SD y el DT, tanto en la fase pre como post tratamiento. Sin embargo, la puntuación encontrada en lectura se acerca en la fase pre-tratamiento y, finalmente, se iguala a la obtenida por el DT, en la fase post tratamiento.
Discusión

Este estudio pretender poner de manifiesto la relación que existe entre las características cognitivas/lingüísticas de un niño con SD y la adquisición y desarrollo del proceso de lectura y escritura. Más específicamente, se pretende conocer la diferencia, en primer lugar, a nivel cognitivo/lingüístico y, en segundo lugar, en el nivel de lectura y escritura entre un preescolar con SD y otro con DT. De igual manera pretende observar el nivel de relación que existe entre el perfil cognitivo/lingüístico y el nivel de lectoescritura, antes y después de un programa de AT.

La ejecución en la prueba de inteligencia llevada a cabo en este estudio (WPPSI), deja patente las diferencias en CI existentes entre el niño con SD y el DT, diferencias ampliamente demostradas en investigaciones y trabajos previos (Pueschel, 2002; Robles, 2007b; Ruiz, 2001) y que han sido descritas por diferentes autores a lo largo de la historia de esta alteración cromosómica común, caracterizada por la discapacidad intelectual. De este modo, en este 
estudio se muestran ejecuciones del niño con SD en las pruebas que evalúan CI (verbal, manipulativo y total) considerablemente inferiores al niño con DT.

Además de la discapacidad intelectual, diversas investigaciones han examinado las diferencias existentes entre preescolares con SD y preescolares de inteligencia media o DT en la ejecución de diferentes tareas, estableciendo así un perfil neuropsicológico especifico (ver, por ejemplo, Calero et al., 2010; Menghini, Constanzo, \& Vicari, 2011). Señalar que los resultados muestran que las mayores diferencias entre ambos niños están dentro de lo que se esperaba según la literatura científica ya que la mayor dificultad en el niño SD se centra en la modalidad auditivo-verbal, tanto en memoria como en procesamiento (planificación verbal) (Buckley \& Perea, 2005; Calero et al., 2010). En esta línea, los resultados de este estudio son congruentes con los hallados en las investigaciones previas señaladas, encontrándose un nivel de ejecución inferior en las distintas pruebas cognitivas y lingüísticas en el preescolar con SD en comparación con el preescolar con DT. Estos resultados sugieren que la discapacidad intelectual conlleva un funcionamiento más enlentecido en la mayoría de las áreas de desarrollo.

Sin embargo, aunque en la evaluación realizada a los 6 meses (fase post) las diferencias de ejecución entre ambos niños en las distintas tareas son bastante apreciables, los resultados sugieren puntuaciones de ganancia logradas por el niño con SD en algunas de las áreas evaluadas que resultan equiparables a las ganancias observadas en el niño control y asociadas al paso del tiempo. En esta dirección, similares puntuaciones de ganancias en la prueba CUMANIN tanto en desarrollo verbal - especialmente asociado a la comprensión- como no verbal así como en el CI verbal ofrecido por la prueba WIPPSI y en menor medida en el CI manipulativo. La mayor estimulación del lenguaje en su componente de comprensión observada en el CUMANIN son coherentes con estudios previos en los que se pone de manifiesto que en SD el proceso de comprensión del lenguaje se encuentra menos afectado. Resaltar que estas puntuaciones de ganancia no se consideran asociadas al programa de intervención dirigido a la mejora de las habilidades de lectoescritura sino, especialmente, al programa de estimulación que mantiene el niño con SD y que se continúa junto al programa en lectoescritura

Las dificultades a nivel cognitivo y lingüístico que presentan los niños con discapacidad intelectual pueden verse reflejadas en el proceso de aprendizaje de la lectoescritura existiendo diferencias en el nivel alcanzado de lectura y escritura. Los resultados obtenidos en el presente estudio muestran que la ejecución tanto en lectura como en escritura previa a la intervención es más deficiente en el caso del niño con discapacidad intelectual. Estos resultados son coherentes y confirman los hallazgos encontrados en investigaciones similares previamente realizadas en las que los alumnos con SD tienen dificultades en el aprendizaje de la lectura y escritura (Idol, 2010).

Tras el programa de lectura y escritura de Troncoso \& del Cerro (2005) llevado a cabo con el niño con SD, mejora de forma importante puntuación tanto en lectura como en escritura. Más específicamente, en lectura se alcanza una ganancia en el niño con SD que iguala su puntuación a la obtenida por el niño con DT. También se observa ganancia asociadas a la escritura en la fase post tratamiento aunque sigue siendo inferior a la puntuación del niño con DT.

Una posible explicación de este importante desarrollo de las habilidades lectoras en el niño con SD puede ser efecto de la interacción entre la mejora en las habilidades lingüísticas y el programa de intervención en el desarrollo de las habilidasde de lectoescritura. La relación entre el desarrollo de habilidades lectoras y el desarrollo del lenguaje ha sido identificada en distintos estudios. Mengoni, Nash \& Hulme (2014) y Naess, Melby-Lervag, Hulme \& Lyster, (2012) concluyen que la mejora en el desarrollo del lenguaje en niños con SD se asocia a una mejora en la pruebas de lectura. En la misma dirección Torppa et al (2016) destacan las relaciones bidireccionales entre la comprensión auditiva y las habilidades lectoras de niños controles cuyo idioma materno se caracteriza por ser transparente en la aplicación de las reglas de conversión grafema-fonema.

Estos resultados ponen de manifiesto varios 
aspectos importantes en el proceso de enseñanza de la lectoescritura. En primer lugar, los efectos diferenciales observados en los procesos de lectura y escritura tras la intervención, sugiere que en la enseñanza-aprendizaje, ambos procesos se pueden trabajar a ritmos distintos. Así, muchos alumnos con SD pueden aprender a leer y escribir a un nivel independiente, debido, principalmente a la complejidad que entraña el proceso de escritura, siendo la lectura un proceso más sencillo (Troncoso\& del Cerro, 2005).

En segundo lugar, muestra que el proceso de aprendizaje de la lectura puede ser relativamente independiente de su madurez intelectual. De forma similar a lo encontrado en este estudio inicial, Klusek et al (2015) hallaron que la adquisición de habilidades lectoras en niños que presentan Síndrome de X frágil (SXF) superaba las expectativas derivadas de sus niveles de desarrollo cognitivo. La reducción en la importancia dada al cociente intelectual en el desarrollo de habilidades lectoras en casos de discapacidad intelectual también podría ser aplicable a otros Síndromes como es el SD (Buckley \& Perea, 2005; Valencia \& Robles, 2017). En general, este tipo de resultados indican que las personas con SD pueden empezar a leer sin esperar a que alcance un cierto grado de madurez intelectual (Robles-Bello \& Calero, 2003)

Por último, los resultados de este estudio muestran cómo un programa de AT, centrado en lectura y escritura, a nivel preescolar, puede estar relacionado con los beneficios que se encuentran en el proceso de aprendizaje de la lectura y escritura. Numerosos autores están de acuerdo con esta afirmación y proponen que la enseñanza de la lectura, principalmente, debe ser temprana e iniciarse antes que en sus compañeros de clase debido a los efectos positivos que conlleva (Robles-Bello \& Calero, 2003). Más específicamente, con la enseñanza de la lectura temprana se pretende que el niño con SD practique lo antes posible de un tipo de metodología motivante que por un lado aproveche los puntos fuertes a nivel cognitivo del SD ya que sus logros suelen ser mejores de lo esperado dadas sus dificultades de aprendizaje (bajo CI), retraso en el habla, lenguaje, memoria de trabajo y capacidades motrices (Robles, 2010). Loveall \&
Conners (2016) relacionan esta fortaleza en la identificación de palabras en niños con SD con el desarrollo en el conocimiento ortográfico específico a las palabras diferenciándolo. Para esos autores, el énfasis en este conocimiento ortográfico específico a la palabra puede ser el producto de programas de enseñanza de la lectura centrados en el reconocimiento de la palabra total y/o de la estrategia adoptada por los niños con SD ante sus dificultades con la recodificación fonológica.

En este proceso de enseñanza también puede ser posible la combinación posterior con una orientación más fonológica dado los resultados prometedores de estudios como el de Lemons et al (2015) quienes adaptando un programa convencional de adquisición de la lectura centraba en habilidades fonológica a las características fenotípicas de niños con SD y con edades entre los 6 y 8 años, obtuvieron una mejora importante en la conciencia fonológica de los niños.

En definitiva, las diferencias que se encuentran en este estudio a nivel cognitivo y psicolingüístico en el niño con SD con respecto al DT pueden ser independientes del nivel de lectura y escritura que se alcance, entre otros motivos, por la participación en un programa de AT, desde edades preescolares. De este modo, a pesar de las dificultades, se puede afirmar que puede existir una positiva evolución en el desarrollo del participante con SD, gracias a la atención y educación durante las etapas tempranas de la vida. Además, cuando los programas escolares son adecuados en su contenido y en el modo de llevarlos a cabo, la mejoría observada en sus niveles de desarrollo se puede mantener elevada en comparación con épocas anteriores. Y esto es tanto más interesante cuando el desarrollo de tales programas no exigen del individuo unos niveles intelectuales muy altos con los que podrán realizarse toda una serie de actividades muy variadas que pueden aprenderse con metodologías adaptadas, como es el caso del método de lectura y escritura de Troncoso \& del Cerro (2005), llevado a cabo en este estudio.

Igualmente es importante señalar las implicaciones prácticas y clínicas de este tipo de intervenciones, ya que si bien las diferencias obtenidas y descritas entre un niño con SD y niños con desarrollo típico son bien conocidas, este 
trabajo aporta la evidencia y la eficacia de un programa de tratamiento en la lectura y escritura. La lectura estimula el enriquecimiento semántico, la capacidad sintáctica y el perfeccionamiento de la pronunciación de las palabras. Es al verlas escritas cuando el niño llega a darse cuenta de que, en su lenguaje ordinario, omite la pronunciación de letras o sílabas. Y al esforzarse en leerlas bien, las articula mejor. No hay un solo lector que no haya mejorado su lenguaje oral en contenido, en articulación y en morfosintaxis (Robles-Bello \& Sánchez-Teruel, 2013). Por otra parte, la afición lectora facilita el enriquecimiento en la exposición de conceptos, en el empleo de términos y expresiones. El lector ve bien escrito lo que tantas veces ha oído mal y no se ha atrevido a pronunciar. Naturalmente, esto exige un ambiente familiar en el que se premie el diálogo, en el que existan claramente "espacios temporales" dedicados a la conversación, por pobre que ésta pueda ser inicialmente. Es entonces cuando el niño, crecientemente familiarizado con lo que lee, encuentra el tiempo y el clima apropiados para expresarlo verbalmente. Aunque es posible que después, en otro ambiente menos familiar, siga prevaleciendo su silencio o su timidez. Pero poco a poco se va venciendo. La lectura, pues, es un apoyo inigualable del enriquecimiento verbal y conversacional (Troncoso \& del Cerro, 2005).

Finalmente resulta conveniente señalar las limitaciones de este estudio, entre las que se destaca, principalmente, la muestra utilizada o el período de tratamiento. En esta línea es conveniente plantear una investigación posterior en la que se pudiese obtener una muestra mayor (tanto SD como DT) para poder generalizar los resultados así como un período de intervención más larga (un curso completo de AT). Además, resulta necesario seguir realizando investigaciones, que pongan de manifiesto las conclusiones de este estudio, más aun teniendo en cuenta la importancia que tienen las habilidades de lectura y escritura para la población con SD. De este modo, la obtención de un nivel aceptable de estos procesos les puede ayudar a participar en una educación más formal, aumentar las oportunidades de empleo, tener acceso a libros y periódicos $\mathrm{y}$, en definitiva, conseguir un mayor grado de autonomía personal, laboral y social.

\section{Referencias}

Aguinaga, G., Armentia, M., Fraile, A., Olangua, P., \& Uriz, N. (2004). PLON-R Prueba de Lenguaje Oral de Navarra revisada de 3 a 6 años. Madrid: TEA.

Angulo, M.C., Gijón, A., Luna, M., \& Prieto, I. (2008). Manual de atención al alumnado con necesidades específicas de apoyo educativo derivadas de Síndrome de Down. Andalucía: Junta de Andalucía. Consejería de Educación.

Bautista, R. (2002). Necesidades educativas especiales. Málaga: Aljibe.

Buela-Casal, G., \& Sierra, J.C. (2002). Normas para la redacción de casos clínicos. International Journal of Clinical and Health Psychology. 2, (3), 525-532.

Buceta, M. J. (2011). Manual de Atención Temprana. Madrid: Síntesis.

Buckley, S., \& Sacks, B.I. (2002). An overview of the development of teenagers with Down Syndrome. Portsmounth, UK: The Down Syndrome Educational Trust.

Buckley, S., \& Perea, J. (2005). Lectura y escritura en alumnos con Síndrome de Down. En Buckley S. \& Perea J (Eds.). Desarrollo del habla y del lenguaje en alumnos con Sindrome de Down (volumen II). Madrid: CEPE.

Calero, M. D., Robles, M. A., \& García, M. B. (2010). Habilidades cognitivas, conducta y potencial de aprendizaje en preescolares con Síndrome de Down. Electronic Journal of Research in Educational Psychology, 8, 87110.

Edgin, J. O., Pennington, B. F., \& Mervis, C. B. (2010). Neuropsychological components of intellectual disability: The contributions of inmediate, working, and associative memory. Journal of Intellectual Disability Research, 54 (5), 406-417.

Galeote M., Soto P., Sebastián E., Rey, R., \& Checa, E. (2012). La adquisición del vocabulario en niños con Síndrome de Down: Datos normativos y tendencias de desarrollo. Infancia y aprendizaje, 35 (1), 111-122.

García, J., Portellano, J. A., \& Díaz, F. (2010). Aspectos neuropsicológicos y hemodinámicos en el Síndrome de Down. Nuevas 
aportaciones. Revista Síndrome de Down, 27, 149-158.

Gough, P. B., \& Tunmer, W. E. (1986). Decoding, reading, and reading disability. RASE: Remedial \& Special Education, 7, 6-10.

Idol, L. (2010). Reading success: Validation of a specialized literacy program. Remedial and Special Education, 31, 97-119.

Klusek, J., Hunt, A. W., Mirrett, P. L., Hatton, D. D., Hooper, S. R., Roberts, J. E., \& Bailey, D. B. (2015). Reading and Phonological Skills in Boys with Fragile X Syndrome. Journal of Autism and Developmental Disorders, 45, 1699-1711.

Krinsky-McHale, S. J., Kittler, P., Brown, W. T., Jekins, E. C., \& Devenny, D. A. (2005). Repetition priming in adults with Williams Syndrome: Age-related dissociation between implicit and explicit memory. American Journal on Mental Retardation, 110, 482-496.

Lemons, C. J., King, S. A., Davidson, K. A., Puranik, C. S., Fulmer, D., Mrachko, A. A., Partanen, J., Otaiba, S. A. \& Fidler, D. J. (2015). Adapting phonological awareness interventions for children with Down Syndrome based on the behavioral phenotype:

A promising approach? Intellectual and Developmental Disabilities, 53, 271-288.

Loveall, S. J., \& Conners, F. A. (2016). Reading skills in Down Syndrome: An examination of orthographic knowledge. American Journal on Intellectual and Developmental Disabilities, 121, 95-110.

Menghini, D., Costanzo, F., \& Vicari, S. (2011). Relationship between brain and cognitive processes in Down Syndrome. Behavior Genetics, 1, 381-393.

Mengoni, S. E., Nash, H. M., \& Hulme, C. (2014). Learning to read new words in individuals with Down syndrome: Testing the role of phonological knowledge. Research in Developmental Disabilities, 35, 1098-1109.

Montero, I., \& León, O.G. (2002). Clasificación y descripción de las metodologías de investigación en Psicología. International Journal of Clinical and Health Psychology. 2, (3), 503-508.

Naess,K., Melby-Lervag, M, Hulme, C., \& Lyster, S. (2012). Reading skills in children with Down syndrome: A meta-analytic review.
Research in Developmental Disabilities, 33, 737-747.

Portellano, J. A., Mateos, R., \& Martínez, R. (1999). CUMANIN. Cuestionario de Madurez Neuropsicológica Infanti. Ed. TEA Ediciones, S.A.

Pueschel, S. M. (2002). Síndrome de Down: Hacia un futuro mejor. Guía para padres. Cantabria: Masson.

Robles-Bello, M. A. (2007). Utilidad de la escala ACFS para población preescolar con Síndrome de Down. Tesis Doctoral, Departamento de Personalidad, Evaluación y Tratamiento Psicológico. Universidad de Granada.

Robles-Bello, M. A. (2010). Respuesta a la lectura de un grupo de niños con Síndrome de Down de un programa de Atención Temprana. Apuntes de Psicología, 28 (3), 367-376.

Robles-Bello, M. A., \& Calero M. D. (2003). Evaluación del Potencial de Aprendizaje de la lectura en Síndrome de Down. Siglo Cero Revista Española sobre Discapacidad Intelectual, 34 (2), 14-26.

Robles-Bello, M. A., \& Sánchez-Teruel, D. (2011). Evaluación e intervención en atención infantil temprana: Hallazgos recientes y casos prácticos. Jaén: Publicaciones de la Universidad de Jaén.

Robles-Bello, M. A., \& Sánchez-Teruel, D. (2013). ¿Qué es el Síndrome de Down? Actualización en Síndrome de Down: Creer para ver. Jaén: Fortalezas Editorial.

Ruiz, E. (2001). Evaluación de la capacidad intelectual en personas con Síndrome de Down. Revista de Síndrome de Down, 18, 8088.

Snowling M. J., Hulme C., \& Mercer R. C., (2002). A deficit in rime awareness in children with Down Syndrome. Reading \& Writing 2, 471-495.

Torppa, M., Georgiou, G. K., sceperierkanen, M.K., s.tem Niemi, P., Poikkeus, A.M., \& Nurmi, J.E. (2016). Examining the Simple View of Reading in a transparent orthography: A longitudinal study from kindergarten to grade 3. Merrill-PalMer Quarterly, 62, 179-206.

Troncoso, M.V \& del Cerro, M.M. (2005). Síndrome de Down: Lectura y escritura. Cantabria: Masson. (Disponible en http://www 
.down21materialdidactico.org/librolectura/ind ex.html).

Valencia, N., \& Robles-Bello, M. A. (2017). Learning potential and cognitive abilities in preschool boys with fragile $\mathrm{X}$ and Down syndrome. Research in Developmental Disabilities 60, 153-161. http://dx.doi.org/10.1016/j.ridd.2016.12.001

van Bysterveldt, A., \& Gillon, G. (2014). A descriptive study examining phonological awareness and literacy development in children with Down Syndrome. Folia Phoniatrica et Logopaedica, 66, 48-57.

Wechsler, D. (1967). Manual for the Wechsler Preschool and Primary Scale of Intelligence. New York University School of Medicine. (Also, distributed by the Psychological Corporation, New York.).

Zulueta, M.I. \& Mollá, M.T. (2006): Programa para la estimulación del desarrollo infantil. Madrid: Cepe. 